\title{
Tuning Chemical Potential Difference across Alternately Doped Graphene p-n Junctions for High-Efficiency Photodetection
}

Li Lin ${ }^{\dagger+,}$, Xiang X X ${ }^{\xi,+}$, Jianbo Yin ${ }^{\dagger}$, Jingyu Sun ${ }^{\dagger}$, Zhenjun Tan ${ }^{\dagger, \perp}$, Ai Leen Koh ${ }^{\|}$, Huan Wang $^{\dagger}$, Hailin Peng ${ }^{*,+}$, Yulin Chen ${ }^{\xi, \#, *}$, Zhongfan Liu ${ }^{*,+}$

${ }^{\dagger}$ Center for Nanochemistry, Beijing Science and Engineering Center for Nanocarbons, Beijing National Laboratory for Molecular Sciences, College of Chemistry and Molecular Engineering, Peking University, Beijing 100871, P. R. China

${ }^{\#}$ Clarendon Laboratory, Department of Physics, University of Oxford, Parks Road, Oxford, OX1 3PU, UK

"Stanford Nano Shared Facilities, Stanford University, Stanford, California 94305, USA

${ }^{\S}$ State Key Laboratory of Low Dimensional Quantum Physics, Collaborative Innovati on Center of Quantum Matter and Department of Physics, Tsinghua University, Beijin g 100084, China

${ }^{\perp}$ Academy for Advanced Interdisciplinary Studies, Peking University, Beijing 100871, China 


\begin{tabular}{|ccc|}
\hline Group & $\begin{array}{c}\text { Chemical potential } \\
\text { difference (eV) }\end{array}$ & Method \\
\hline Zhongfan Liu ${ }^{1}$ & 0.20 & CVD-grown i-n junction \\
Philip Kim ${ }^{2}$ & 0.30 & Electric filed effect \\
Phaedon Avouris & & Electrostatic substrate engineering \\
Timm Lohmann & & PMMA doping \\
Bin Yu & 0.26 & Local electrical stress \\
Klaus Kern & 0.12 & Absorbent doping \\
This work & 0.35 & CVD-grown p-n junction \\
\hline
\end{tabular}

Table S1: Reported chemical potential differences of graphene p-n junctions 


\section{Supplementary Figures:}

a
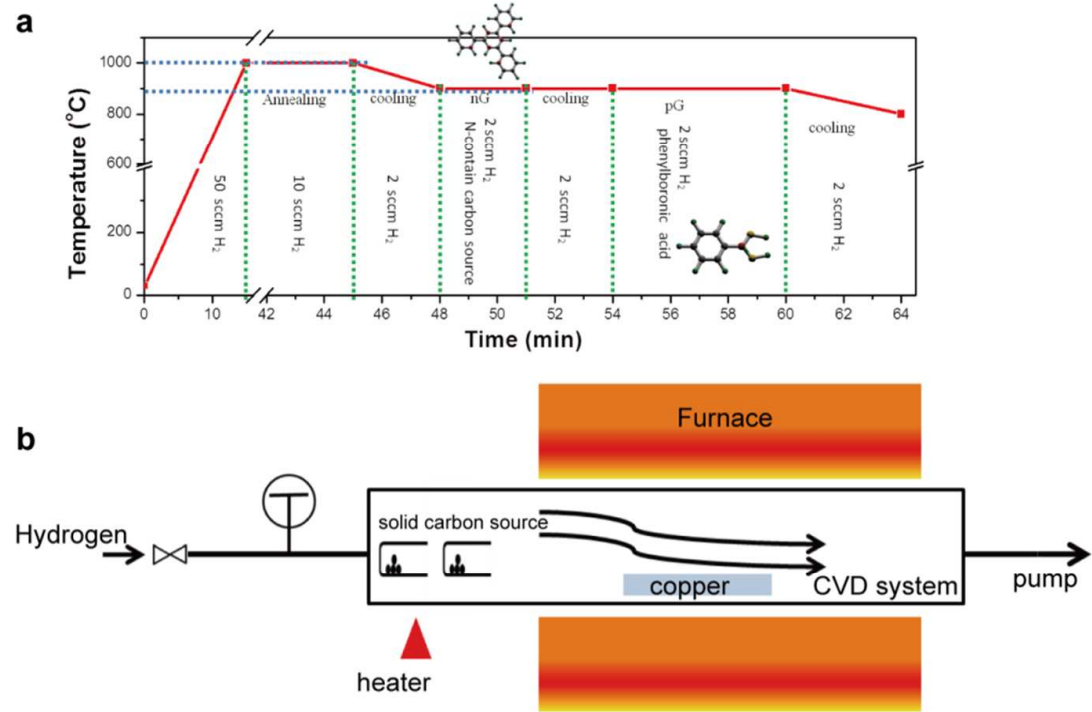

Figure S1. Drawing of growth protocol and CVD system for growing alternately BNG p-n junction. (a) Growth procedure of alternately BNG p-n junction. The flow of hydrogen varies from $0.3 \mathrm{sccm}$ to $50 \mathrm{sccm}$. The growth time of NG varies from 3 $\min$ to $20 \mathrm{~min}$. The growth time of $\mathrm{BG}$ varies from $5 \mathrm{~min}$ to $30 \mathrm{~min}$. After the nucleation of NG inlands, $100 \mathrm{sccm}$ Ar was introduced to the system to clean the remaining nitrogen-containing carbon source. (b) Drawing of the low-pressure CVD system, where the solid carbon source is positioned upstream $40 \mathrm{~cm}$ (phenylboronic acid) and $30 \mathrm{~cm}$ (2,4,6-Tri(2-pyridyl)-1,3,5-triazine) away from the hot center. The heater is a heating tape with temperature set to $150{ }^{\circ} \mathrm{C}$ and $120{ }^{\circ} \mathrm{C}$ for evaporation of 2,4,6-Tri(2-pyridyl)-1,3,5-triazine and phenylboronic acid, respectively. $^{7}$ 

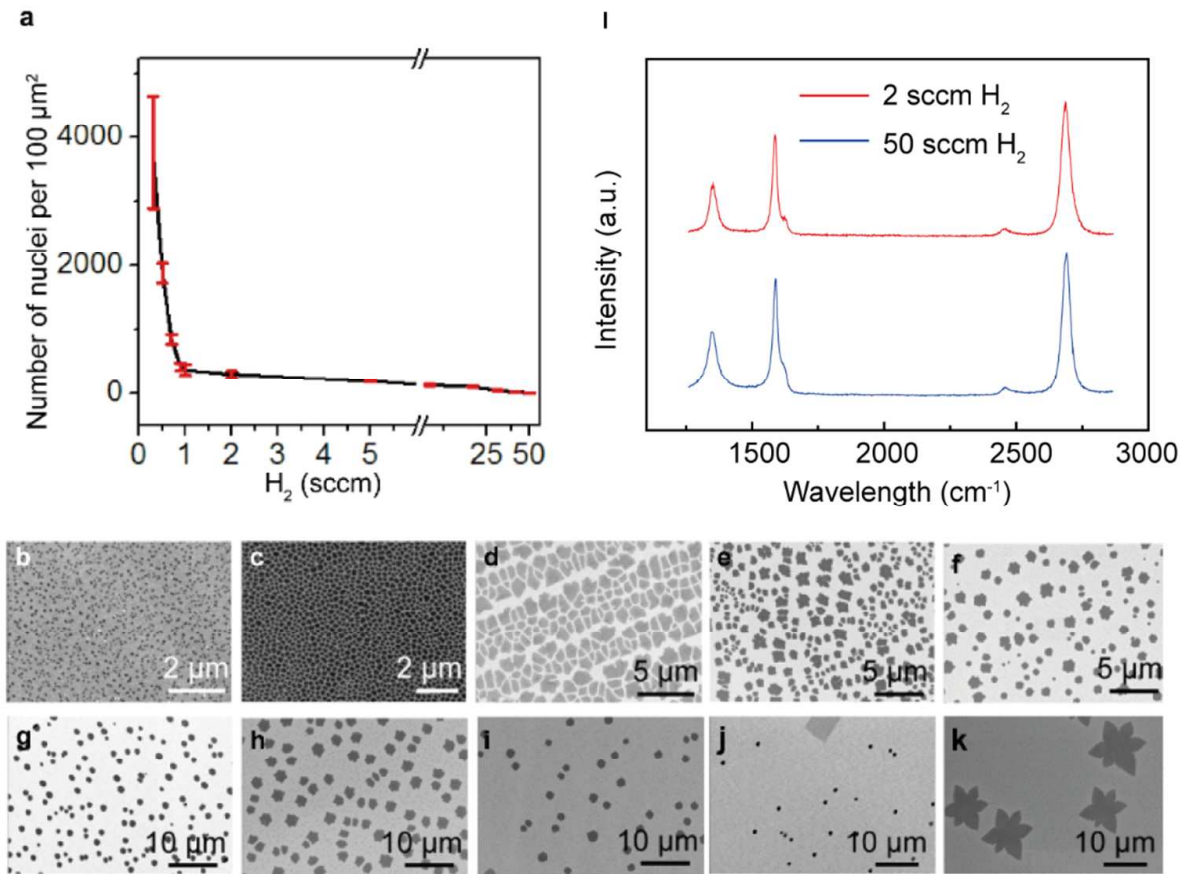

Figure S2. The nucleation density of NG islands as a function of flow rate of $\mathbf{H}_{2}$.

(a) The nucleation density of NG islands as a function of flow rate of $\mathrm{H}_{2}$. (b-k) The SEM images of NG islands grown on copper foils. The flow rate of $\mathrm{H}_{2}$ is $0.3,0.5,1,2$, $5,10,20,30,40,50 \mathrm{sccm}$, respectively. (1) Raman spectra of the NG grown with the $\mathrm{H}_{2}$ flow rate of $2 \mathrm{sccm}$ (red) and $50 \mathrm{sccm}$ (blue). Note that, the hydrogen flow function as not only the reducing gas, but also the carrier gas for transporting the nitrogen or boron containing gas. Consequently, the flow rate of $\mathrm{H}_{2}$ might also influence the doping level of the as-formed graphene through influencing the concentration of nitrogen specie on the copper surface ${ }^{8}$. However, demonstrated in Fig. S21, the D band intensity of the NG films formed with $2 \mathrm{sccm}$ and $50 \mathrm{sccm} \mathrm{H}_{2}$ are equal, indicating the same doping level. In this regard, the doping level of as-formed graphene is mainly determined by the growth temperature through controlling the competition between formation of $\mathrm{C}-\mathrm{N}$ or $\mathrm{C}-\mathrm{B}$ bond and the $\mathrm{C}-\mathrm{C}$ bond. 

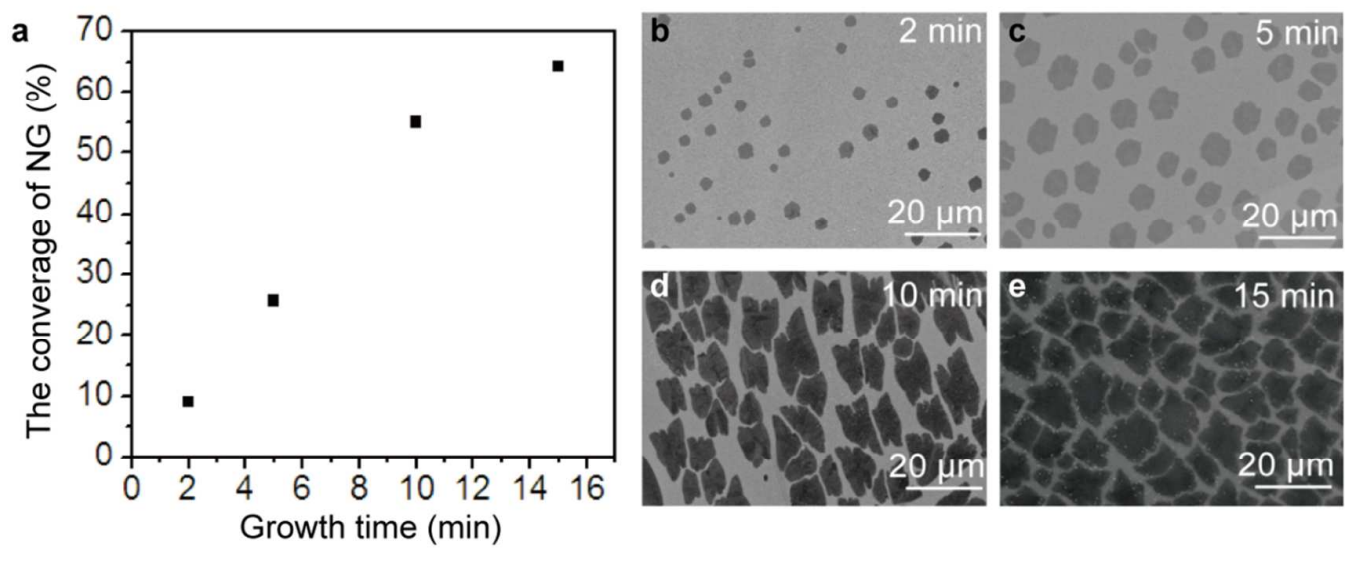

Figure S3. The coverage of NG as a function of growth time of NG nuclei. (a) The coverage of NG as a function of growth time of NG nuclei. (b-e) SEM images of NG inlands grown on copper, which display a dark contrast. The growth time of NG islands is $2,5,10,15 \mathrm{~min}$, respectively. The flow rate of $\mathrm{H}_{2}$ is $10 \mathrm{sccm}$. 

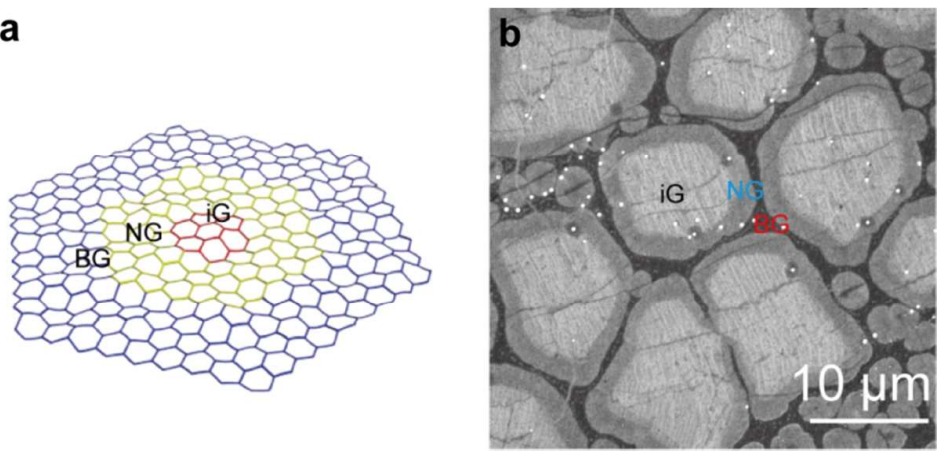

Figure S4. The growth of i-n-p junction through multiple modulation cycles. (a) Schematic diagram of i-n-p junction. After the intrinsic graphene was formed using $\mathrm{CH}_{4}$ (The flow rate: $2 \mathrm{sccm} \mathrm{CH}_{4}$ and $2 \mathrm{sccm} \mathrm{H}_{2}$ and growth time: $30 \mathrm{~s}$ ), the 2,4,6-Tri(2-pyridyl)-1,3,5-triazine was introduced into CVD system for in-plane epitaxial growth of NG from the edge of intrinsic graphene inlands (Growth time: 2 min). Subsequently, phenylboronic acid was transported into the system for in-plane epitaxial growth of BG from the edge of NG shell. Such growth result would justify the epitaxial growth mechanism of modulation-doped method. ${ }^{1}$ (b) The corresponding SEM image of in-plane i-n-p junction. 


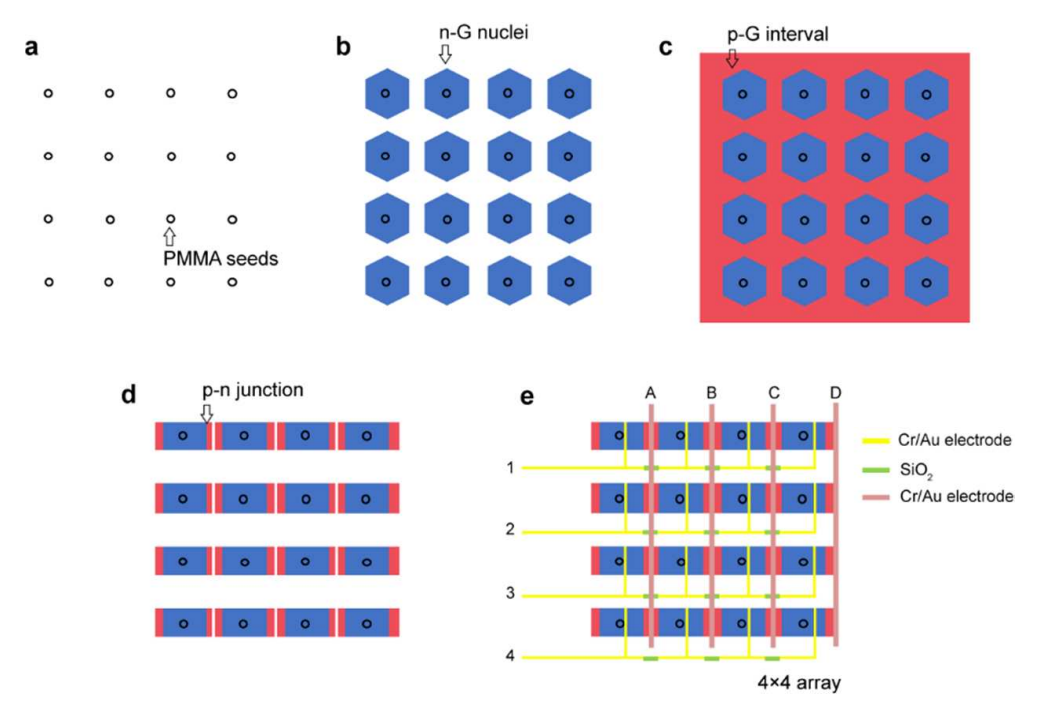

Figure S5. Schematic illustrations of potential application of large-area grown p-n junctions in array photodetector. Once PMMA seeds for the nucleation of nitrogen-doped grains were specifically predefined, the subsequent growth of $p-n$ junction would be precisely template (a-c). ${ }^{1}$ Subsequently, the p-n junctions were isolated by using a PMMA etch mask from Electron Beam Lithography (EBL) and Reactive Ion Etching (RIE) $\mathrm{O}_{2}$ etching (d). Finally, using EBL to design a PMMA mask, the p-n junction can be contacted with electrodes by using the electron-beam evaporator and followed by a standard metal lift-off technique. Note that, $\mathrm{SiO}_{2}$ layer should be deposited between the two electrode to avoid the short circuit (e). 

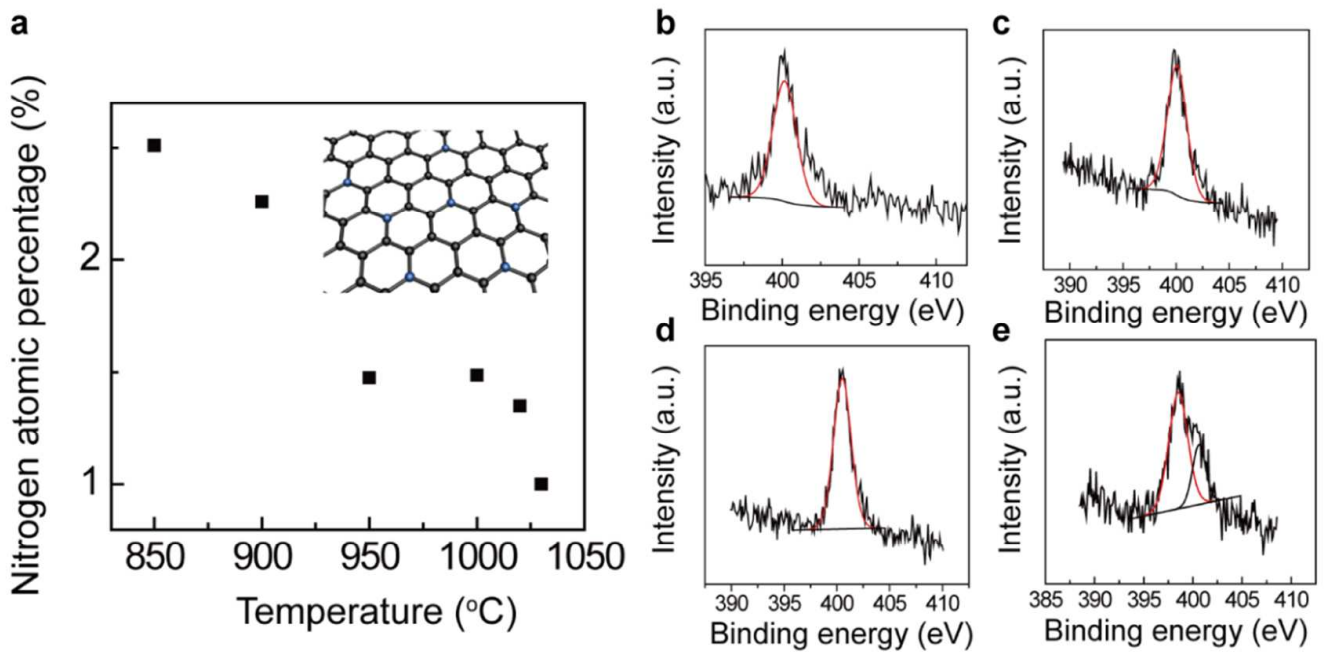

Figure S6. The temperature dependence of the doping concentration and doping

type of as-formed NG flims. (a) The doping concentrations of NG films from the XPS results as a function of growth temperature. The NG films are solely nitrogen-doped graphene with full coverage of the copper. Note that, the characterization was performed on nitrogen-doped graphene, which is transferred onto $\mathrm{SiO}_{2} / \mathrm{Si}$ substrate four times to form multilayer, in order to enhance the XPS signal. (b-e) XPS spectra of solely nitrogen-doped graphene grown at $1000{ }^{\circ} \mathrm{C}(\mathrm{b}), 950{ }^{\circ} \mathrm{C}(\mathrm{c})$, $900^{\circ} \mathrm{C}(\mathrm{d})$ and $850^{\circ} \mathrm{C}$. The atomic percentage of nitrogen is estimated by taking the peak area ratio of the $\mathrm{N} 1 \mathrm{~s}$ at $400.7 \mathrm{eV}$ to the $\mathrm{C} 1 \mathrm{~s}$ at $284.8 \mathrm{eV}$ after considering the atomic sensitivity factors. The $\mathrm{N} 1 \mathrm{~s}$ peak of $\mathrm{NG}$ film fabricated at $850{ }^{\circ} \mathrm{C}$ has two components at 401.0 and $388.7 \mathrm{eV}$, which can be assigned to graphitic- $\mathrm{N}$ and pyridinic-N, respectively. ${ }^{9}$ And the NG films formed at tempature higher than $900{ }^{\circ} \mathrm{C}$ exhibit single peak at around $401.0 \mathrm{eV}$, which can be assigned to substitutional doping type. Such observations indicate that the cofiguration of nitrogon atoms in graphene lattics is changed from graphitic-N to a combination of graphitic-N and pyridinic-N, when growth temperature is lower than $900{ }^{\circ} \mathrm{C}$. 


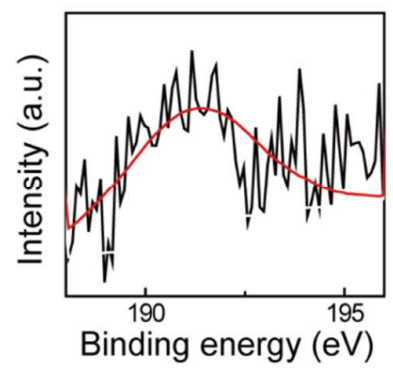

Figure S7. The doping concentration and doping type of BG film. XPS spectrum of solely boron-doped graphene grown at $900{ }^{\circ} \mathrm{C}$. The atomic percentage of boron is estimated by taking the peak area ratio of the $\mathrm{B} 1 \mathrm{~s}$ at $191.2 \mathrm{eV}$ to the $\mathrm{C} 1 \mathrm{~s}$ at $284.8 \mathrm{eV}$ after considering the atomic sensitivity factors. The characterization was performed on $\mathrm{f}$ boron-doped graphene, which is transferred onto $\mathrm{SiO}_{2} / \mathrm{Si}$ substrate four times to form multilayer, in order to enhance the XPS signal. The configuration of boron in the graphene skeleton was confirmed to be substitutionally doping type by the bind energy of B 1s peak, which suggests that the main bonding form of boron atoms should be $\mathrm{BC}_{3}{ }^{7,10}$ It is worthy to be noted that, the boron atom is hard to be detected by XPS at relatively low concentration due to its lower sensitivity factor than nitrogen. 

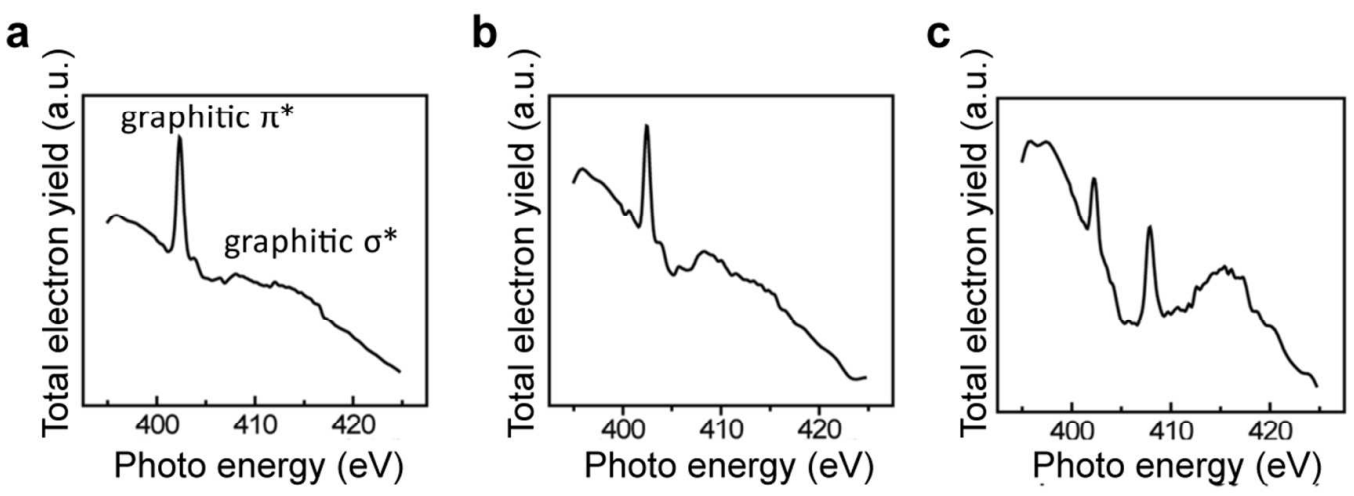

Figure S8. The XANES characterization of doping configuration of nitrogen in NG formed at different temperatures. (a-c) XANES spectra of solely nitrogen-doped films grown at $950{ }^{\circ} \mathrm{C}$ (a), $900{ }^{\circ} \mathrm{C}(\mathrm{b})$, and $850{ }^{\circ} \mathrm{C}$ (c). Note that, the characterization was performed on nitrogen-doped graphene, which is transferred onto $\mathrm{SiO}_{2} / \mathrm{Si}$ substrate four times to form multilayer, in order to enhance the XPS signal. The peaks at $400 \mathrm{eV}$ and $408 \mathrm{eV}$ can be assigned to graphitic $\pi^{*}$ absoption and graphitic $\sigma^{*}$ absoption. In contrast, the peak aroud the $415 \mathrm{eV}$ belongs to the pyridinic-N. These observations indicate that the doping type of nitrogen doped graphene would changed when growth temperature lower than $900{ }^{\circ} \mathrm{C}$, resonating with XPS results. ${ }^{11}$ 


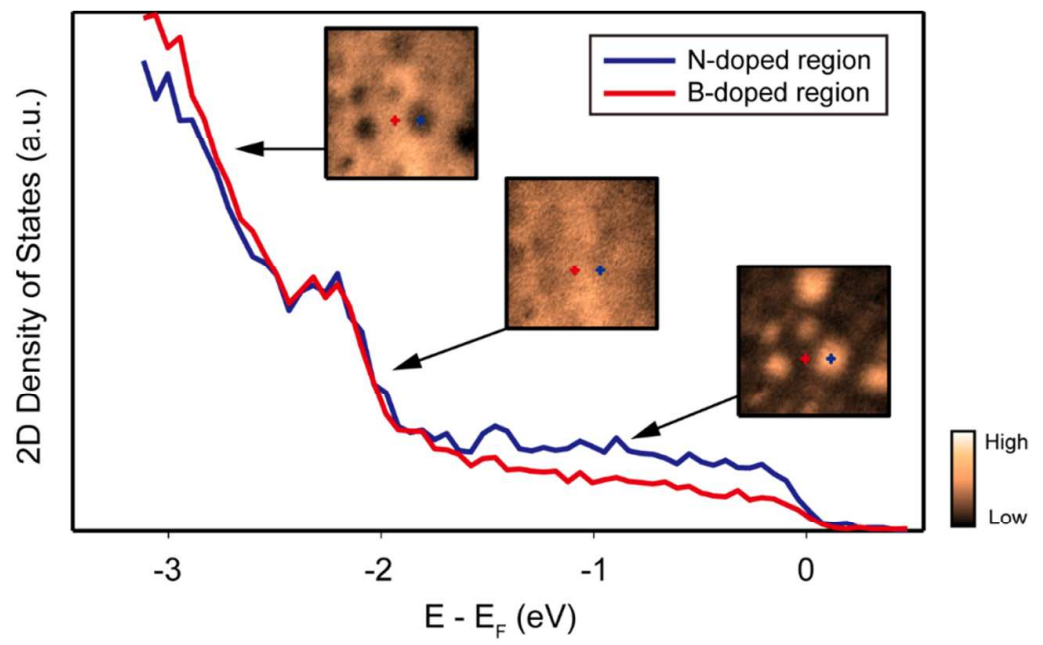

Figure S9. 2D contrast between $\mathbf{N}$-doped and B-doped regions. The contrast of spatial mapping from -1 to $-5 \mathrm{eV}$ basically arises from the band of copper, on which graphene p-n junctions are grown. We focus on the density of state (DOS) originated from the graphene (about $0 \sim 1.5 \mathrm{eV}$ below the Fermi level) and anticipate a contrast between different domains with different doping level. In practice, the graphene DOS contrast is hard to observe because it is based on the two-dimensional DOS (a 2D cut in the momentum space) that we map out the whole spatial morphology. 3D DOS mapping is theoretically feasible, but it takes too much time to map out the 3D DOS of each pixel. A more efficient way to distinguish different domains is turning to the DOS of the copper substrate, the band of which is smooth within several eVs below the Fermi level. The Figure S9 presents the typical 2D DOS of regions with different dopants in the ARPES experiments. The contrast arises from varied transmission ability of the photoelectron emitting from the copper through the graphene sheets with different dopants. 

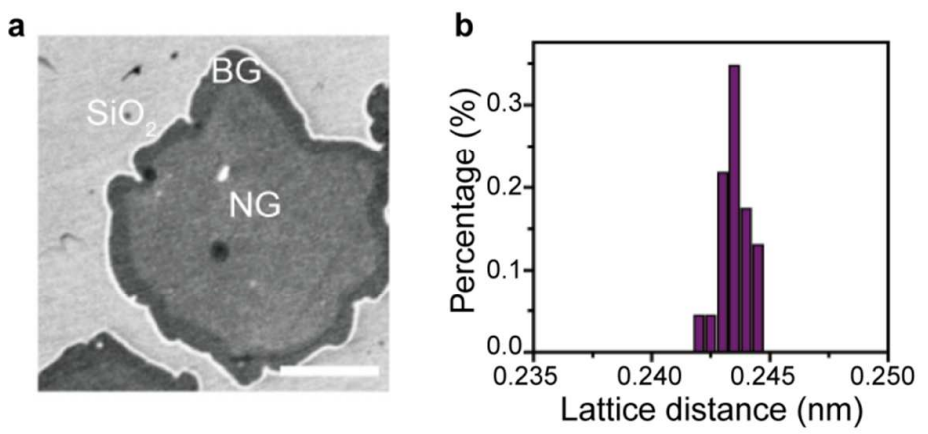

Figure S10. Structural characterization of as-grown graphene p-n junction. (a) SEM image of a discrete alternately BNG p-n junction grain transferred onto $\mathrm{SiO}_{2} / \mathrm{Si}$. Scale bar, $2 \mu \mathrm{m}$. (b) Histogram of the distribution of graphene lattice distance obtained from SAED patterns within the entire grain. The histogram reveals that the average lattice distance is $\sim 2.43 \AA$. 

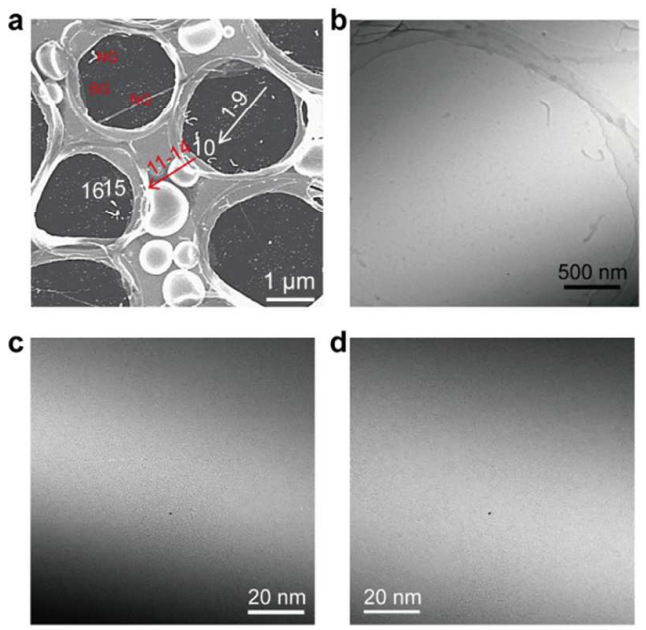
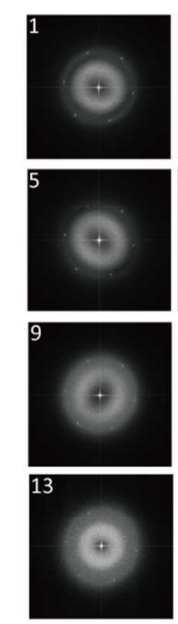
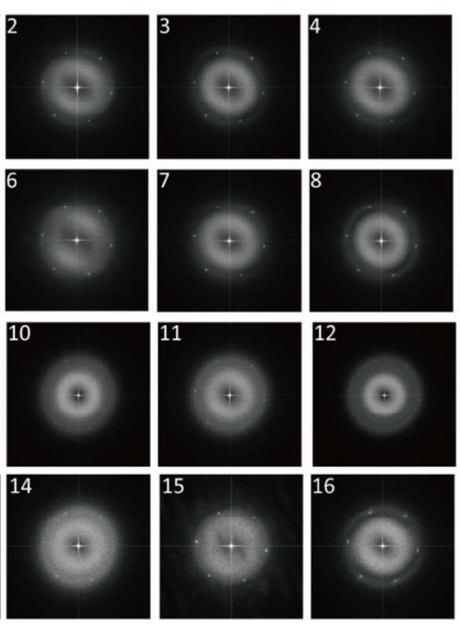

Figure S11. TEM and HRTEM characterizations of alternately BNG p-n

junction. (a) The SEM image of a continuous graphene film consisting of alternately BNG p-n junction transferred onto micrograte TEM grid with hall marks. The NG islands and BG intervals have slight contrast from the SEM image, fortunately due to the preference of the polymethyl methacrylate (PMMA) residual to stay on the NG nuclei, which would provide a nonuniform contrast for locating $\mathrm{p}-\mathrm{n}$ junction. In addition, HRTEM characterizations were performed at the positions marked in SEM image. (b) Low-magnification TEM image of alternating BNG p-n junction film at the same position shown in (a). (c,d) Representative HRTEM images of BG (c) and NG (d) portion of the alternately BNG p-n junction marked in (a). (e) Fourier transformation (FT) pattern of the HRTEM images taken from the postions marked in (a). Note that, the HRTEM images of the $100 \mathrm{~nm} \times 100 \mathrm{~nm}$ areas are taken from the center of NG nuclues to the BG interval sequentially, thus, the NG portion, p-n junction, and PG portion were inclueded. The nealy same orientation of FT patterns obtained from HRTEM image of the NG portion, p-n junction, and PG portion indicates single-crystalline character of alternately BNG p-n junction, which is consistent with the SAED results in Figure. 3. The alternately BNG p-n junction was grown at $900{ }^{\circ} \mathrm{C}$. 

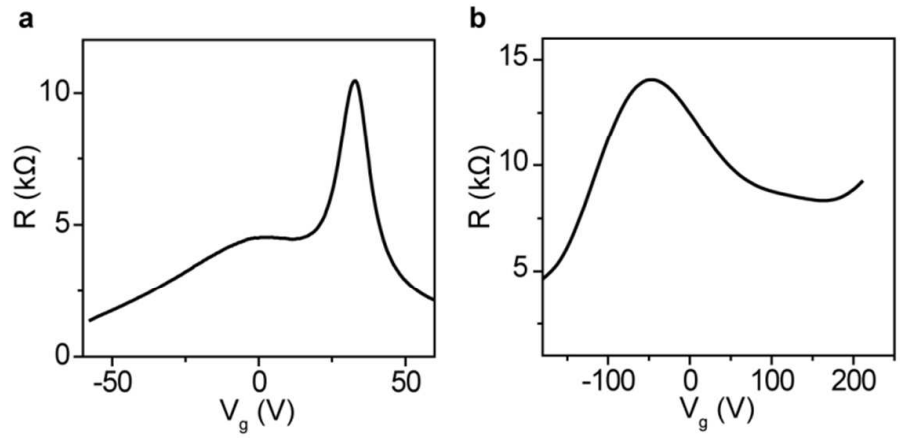

Figure S12. Transfer curves of devices of alternately BNG p-n junction. $(a, b)$ Transfer curves of devices of alternately BNG p-n junction, where the samples were grown at $1000{ }^{\circ} \mathrm{C}(\mathrm{a}), 900{ }^{\circ} \mathrm{C}(\mathrm{b})$. It is well established that graphene $\mathrm{p}$-n junction would produce a curve with two Dirac points and the work function difference across the p-n junction could be calculated from the distance between the two Dirac points. Additionally, we could further extract carrier mobilities near each Dirac point from these curves. In this regard, the chemical potential differences of alternately BNG p-n junctions are $700 \mathrm{meV}, 353 \mathrm{meV}$ and $270 \mathrm{mev}$ for $900 \mathrm{PN}, 950 \mathrm{PN}$ and $1000 \mathrm{PN}$, respectively. The carrier mobilities of the nitrogen-doped portion are $400 \mathrm{~cm}^{2} \mathrm{~V}^{-1} \mathrm{~s}^{-1}$, $640 \mathrm{~cm}^{2} \mathrm{~V}^{-1} \mathrm{~s}^{-1}, 1,050 \mathrm{~cm}^{2} \mathrm{~V}^{-1} \mathrm{~s}^{-1}$ for $900 \mathrm{PN}, 950 \mathrm{PN}$ and $1000 \mathrm{PN}$, respectively. The carrier mobilities of the boron-doped portion are $150 \mathrm{~cm}^{2} \mathrm{~V}^{-1} \mathrm{~s}^{-1}, 1075 \mathrm{~cm}^{2} \mathrm{~V}^{-1} \mathrm{~s}^{-1}, 1,900$ $\mathrm{cm}^{2} \mathrm{~V}^{-1} \mathrm{~s}^{-1}$ for $900 \mathrm{PN}, 950 \mathrm{PN}$ and $1000 \mathrm{PN}$, respectively. 


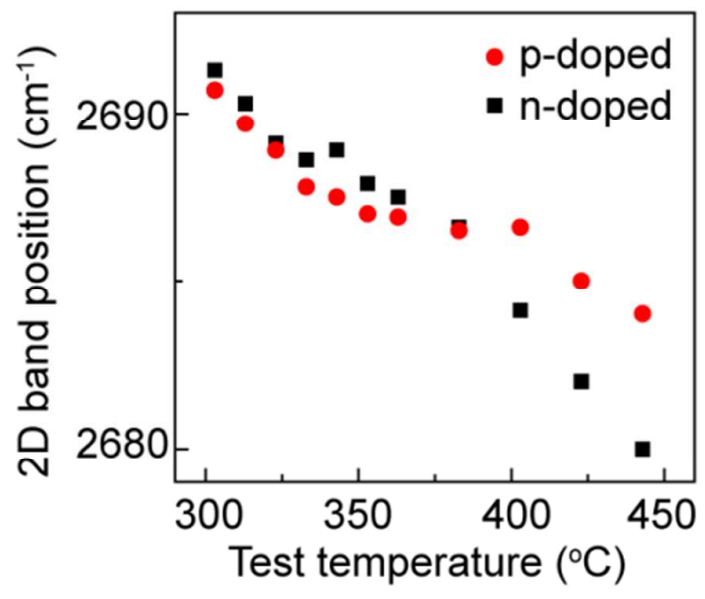

Figure S13. The 2D band position of the nitrogen-doped graphene (black) and boron-doped graphene (red) as function of the test temperature. The sample is heated at an elevated temperature and simultaneously characterized by Raman spectroscopy to probe the change of the doping level with the temperature. Note that, the high-temperature treatment would reduce the doping effect of the absorbed oxygen and water to get a clean surface, which would be reflected by the shift of the 2D band position. The corresponding shifts are different for boron $\left(7 \mathrm{~cm}^{-1}\right)$ and nitrogen (12 $\mathrm{cm}^{-1}$ ) doped graphene, clearly justifying the nonuniform doping effect of absorbed oxygen and water on each portion. Clearly the p-doping effect of the transfer-related charged impurities on n-doped portion is stronger than the p-doped portion of the $p-n$ junction. This is because the difference interaction between absorbent (water and oxygen) with electron or hole doped graphene. Demonstrated in Fig. S12, such non-uniform doping effect can be reduced by high-temperature treatment or in the vacuum. 


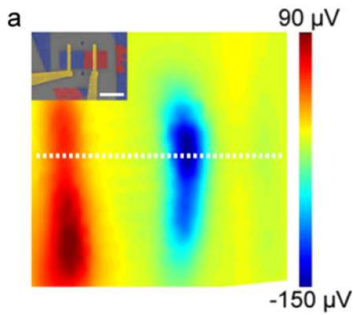

d electrode1

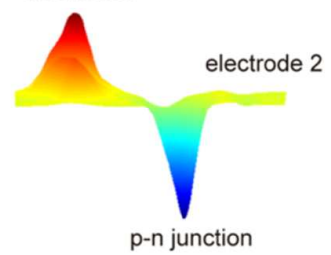

b

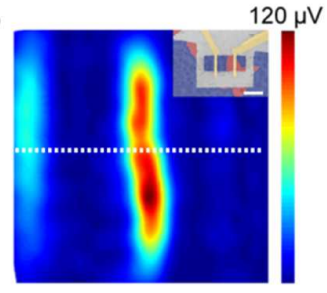

e

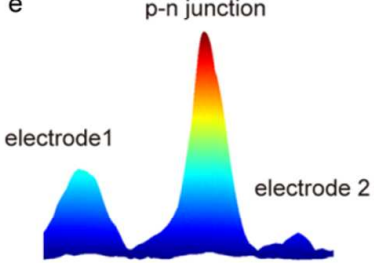

c
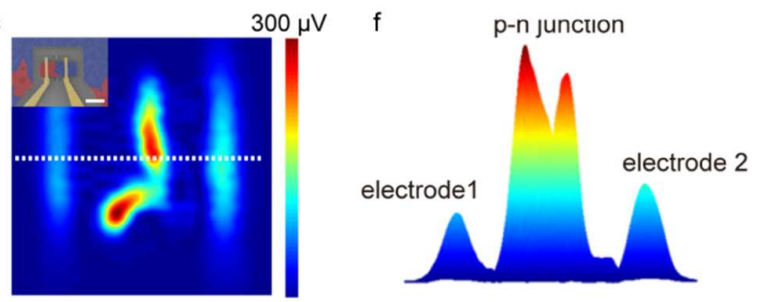

Figure S14. The photovoltage mapping of devices of the alternately BNG p-n junction. (a-c) The photovoltage mapping of the alternately BNG p-n junction devices. The samples were grown at $900{ }^{\circ} \mathrm{C}$ (a), $1000{ }^{\circ} \mathrm{C}(\mathrm{b}), 950{ }^{\circ} \mathrm{C}$ (c), respectively. Inset: SEM images of measured devices, with NG and PG portions false coloured into blue and red, respectively. Scale bar, $5 \mu \mathrm{m}$. (d-f) The corresponding 3-D view of the scanning photovoltage image of the devices. Note tha,t the observation in (c) shows that the scanning photovoltage results can reflect the corresponding morphology of the alternately BNG p-n junction, verifying the prominent generation of photovoltage at the $\mathrm{p}-\mathrm{n}$ junction. 
a

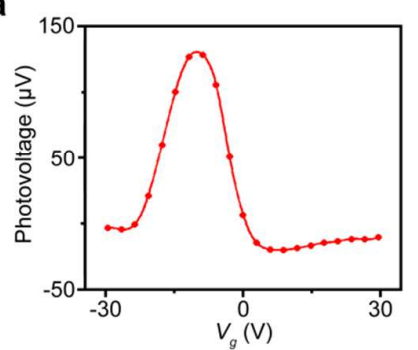

b

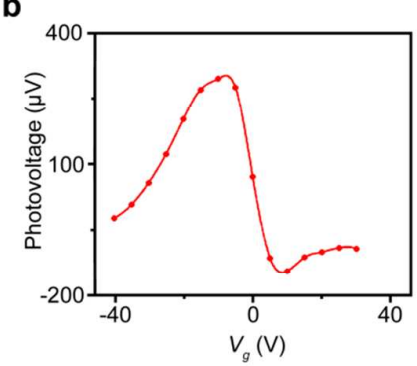

C

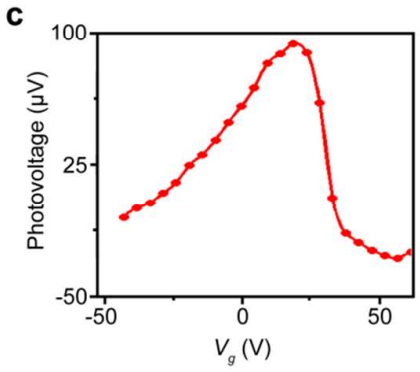

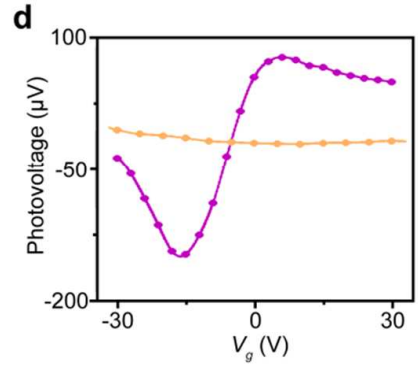

e

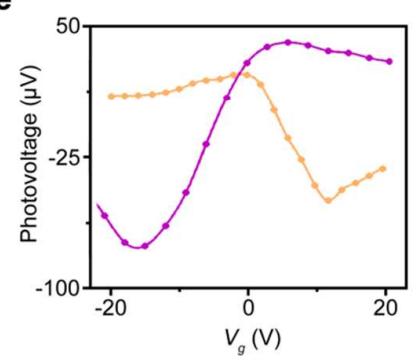

$\mathbf{f}$

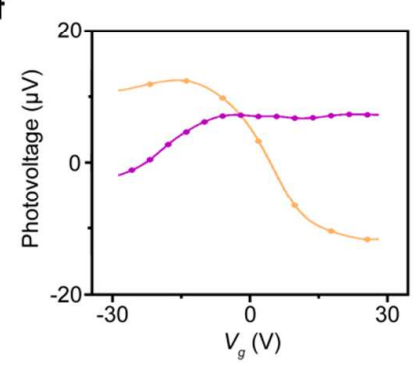

Figure S15. Photovoltage versus applied gate voltage when the laser is located at metal contacts and p-n junction. (a-c) Photovoltage versus applied gate voltage when the laser is located at p-n junction. Note that, the devices probed here are the identical devices in Fig. 4 and Figs. S12a and b. The corresponding alternately BNG p-n junctions are formed at different temperature at $1000{ }^{\circ} \mathrm{C}(\mathrm{a}), 950^{\circ} \mathrm{C}(\mathrm{b}), 900^{\circ} \mathrm{C}$ (c), respectively. (d-f) Photovoltage versus applied gate voltages when the laser is located at metal contacts. The purple (orange) line corresponds to the photovoltage generated when the spot is located at electrode/BG (electrode/NG) area. The photovoltage at the p-n junction changes polarity twice, which indicates that PTE dominates the photovoltage generation. ${ }^{12,13}$ In contrast, the photovoltage at metal contract changes polarity only once, verifying the dominance of PV mechanism in photoconversion process. $^{14}$ 


\section{Experimental details}

Alternately BNG p-n junction growth and transfer. Large-area alternately BNG p-n junction was grown on annealed copper foil loaded inside a low-pressure CVD system. The 2,4,6-Tri(2-pyridyl)-1,3,5-triazine $\quad(99 \%$ purity, J\&K Scientific) and phenylboronic acid (99\% purity, J\&K Scientific) was used as the precursor for the synthesis of nitrogen-doped and boron-doped portions, respectively. The as-grown graphene was transferred onto silicon wafer covered with silicon oxide using PMMA-assisted wet or dry method.

Characterization of mosaic graphene. The morphology and structure of the as-grown alternately BNG p-n junction were characterized with the aid of optical microscopy (Olympus BX51), SEM (Hitachi S-4800, acceleration voltage 5-30 kV) and Raman spectrum (Horiba, LabRAM HR-800, 514 laser wavelength, $\times 100$ objective). The

graphene grains and films on TEM grid was characterized by TEM (FEI Tecnai T20, acceleration voltage $200 \mathrm{kV}$ ). Aberration-corrected TEM studies were performed using an FEI 80-300 Environmental Titan operated in monochromated mode at $80 \mathrm{kV}$. The element analysis of graphene on copper was performed by using AES (PHI Quantera SXM) and XPS (Kratos Analytical AXIS-Ultra with monochromatic Al Ka X-ray). XANES experiments were performed at the Soft X-ray Spectroscopy station and Photoelectron Spectroscopy station of the Beijing Synchrotron Radiation Facility (BSRF).

Device fabrication. Alternately BNG p-n junction was first transferred onto a silicon substrate with silicon oxide as dielectric layer. SEM was used to identify p-n junction from single domain. Standard EBL (STRATADB235, FEI) was carried out to define micro patterns. Designed graphene strips were shaped by plasma etching. Afterward, bilayer metal electrodes $\left(\begin{array}{lllllll}0.5 & \mathrm{~nm} & \mathrm{Cr} / & 50 & \mathrm{~nm} & \mathrm{Au}\end{array}\right)$ were deposited by thermo/e-beam/thermo evaporation (UNIVEX 300, Leybold Vacuum) in one batch. The device was lifted-off by acetone and washed with isopropanol. Finally, it was blow dried with nitrogen gas.

ARPES experiments. The Nano-ARPES experiments were performed on the 
spectromicroscopy beamline at the Elettra. Photons at $74 \mathrm{eV}$ were focused through a Schwarzchild objective to obtain a submicron size spot, enabling a submicron spatial resolution. The sample was first annealed at $350{ }^{\circ} \mathrm{C}$ for 20 mins and kept at about 70 $\mathrm{K}$ with an ultra-high vacuum $\left(10^{-10} \mathrm{mbar}\right)$ during the experiment. The sample is mounted on a micron-resolved XYZ scanning stage, and the electron energy analyzer mounted on two-direction goniometer to achieve angular resolution. The sample was adjusted to the focus plane of the analyzer. By choosing a proper range of binding energy, which optimized the contrast of different domains, and measuring the cumulative electronic distribution within the range pixel by pixel, a $2 \mathrm{D}$ image of morphologic features was obtained. Fix the position of the sample stage, and rotate the analyzer to collect photoelectrons from a continuous series of angles, and the local electronic band structure of a fixed point can be measured.

Transport measurement. A semiconductor analyzer (Keithley, SCS-4200) was used to measure the electrical properties of alternately BNG p-n junction with Keithley 6517A providing gate bias from $-200 \mathrm{~V}$ to $210 \mathrm{~V}$. All the measurements were carried out at room temperature.

Photovoltage measurement. The photoelectrical measurement was performed by a scanning photocurrent microscopy. A focused $532 \mathrm{~nm}$ laser spot $(\sim 1 \mu \mathrm{m}, \sim 250 \mu \mathrm{W})$ was used to excite photocarriers. The chopper-modulated $(\sim 1 \mathrm{kHz})$ laser beam was focused to $\sim 1 \mu \mathrm{m}$ on the device using $\mathrm{X} 100$ objective and the photovoltage signals were then measured using pre-amplifier. When scanning the laser spot over the device, the induced photovoltage signals and beam positions were recorded and displayed simultaneously with the assistance of a computer, which communicated with lock-in amplifier and motorized stage (with device on it). A voltage source (Keithley 2400) was used to apply the gating voltage. All the electrical and photoelectrical measurements were performed in air at room temperature.

Detailed procedure for alternately BNG p-n junction growth. Commercially available copper foil (Alfa-Aesar \#46365, \#46986) was electrochemically polished to clean and 
flatten the surface, before loaded into tube furnace (Lindberg Blue M HTF55667C, 1-inch quartz tube). Diagram of the temperature profile adopted was presented in Fig. S1a. The solid carbon sources were positioned upstream $40 \mathrm{~cm}$ (phenylboronic acid) and $30 \mathrm{~cm}$ (2,4,6-Tri(2-pyridyl)-1,3,5-triazine) away from the hot center. The growth process of alternately BNG p-n junction at $900{ }^{\circ} \mathrm{C}$ is as following:

1. After rapid heating up to $1000{ }^{\circ} \mathrm{C}$ in under a $\mathrm{H}_{2}$ flow of $100 \mathrm{~cm}^{3}$ per min $(\mathrm{sccm})$, copper foil was annealed for 20 min to reduce surface oxides and increase grain size.

2. The temperature gradually dropped to $900{ }^{\circ} \mathrm{C}$ for growth of nitrogen-doped graphene. The 2,4,6-Tri(2-pyridyl)-1,3,5-triazine was heated with a heating tape to $150{ }^{\circ} \mathrm{C}$ for the gradual sublimation, and $2 \mathrm{sccm} \mathrm{H}_{2}$ as carrier and reaction gas was introduced to transport the 2,4,6-Tri(2-pyridyl)-1,3,5-triazine downstream to the copper at the hot center with a corresponding pressure of $6 \mathrm{~Pa}$. The average domain size of as-formed nitrogen-doped nuclei is around $10 \mu \mathrm{m}$ with an interval of $10 \mu \mathrm{m}$

3. Then the vessel was purged with large flow of argon $(\sim 100 \mathrm{sccm})$ for 3 minutes, during which the temperature kept at $900{ }^{\circ} \mathrm{C}$.

4. The phenylboronic acid was heated with a heating tape to $120{ }^{\circ} \mathrm{C}$ for the gradual sublimation, and $2 \mathrm{sccm} \mathrm{H}_{2}$ as carrier and reaction gas was introduced to transport the phenylboronic acid downstream to the copper at the hot center with a corresponding pressure $6 \mathrm{~Pa}$. Note that $2 \mathrm{~min}$ of growth of boron-doped graphene is enough for discrete graphene domain with edge width of $\sim 1 \mu \mathrm{m}$ and 15 min is sufficient for full coverage.

5. After shutting down the heating tap, the sample was quickly cooled to room tempeature in $20 \mathrm{~min}$.

6. The isolated grains or continuous films consisting of alternately BNG p-n junctions were transferred onto dielectric substrates, $\mathrm{SiO}_{2} / \mathrm{Si}$, using PMMA-assisted wet or dry method for characterizations. After transfer, the sample is ready for SEM 
(HITACHI, S-4800), AES (PHI Quantera SXM), XPS (Kratos Analytical AXIS-Ultra with monochromatic Al K $\alpha$ X-ray), Raman spectrum (HORIBA, LabRAM HR800,) and TEM (FEI, T20) characterizations.

Mobility Extraction. Field-effect mobilities were extracted from the transconductance, $\mathrm{g}_{\mathrm{m}}$, as follows,

$$
\mu=\frac{g_{m} L}{C_{o x} W V_{d s}}=\frac{d I_{d s}}{d V_{g s}} \frac{L}{W C_{o x} V_{d s}}
$$

where $\mathrm{C}_{\mathrm{ox}}$ is the gate capacitance per unit area.

Calculation of the photovoltage generation in graphene $p$-n junction. The mechanism of the photocurrent generation in graphene has been discussed in literatures previously reported. ${ }^{1,12,15-18}$ To deduce and estimate the magnitude of photovoltage generated in our graphene p-n junction, we refer the model in ref. 15 based on the our device and testing parameters of photovoltage measurement. Here photovoltage generated by both PTE and PV are discussed, and we show that in our graphene p-n junction, PTE prevails over PV by several orders of magnitude.

For PTE, we firstly start by the classical Mott Formula, ${ }^{18}$ which gives the general Seebeck coefficients of semiconductors:

$$
S=-\frac{\pi^{2} k_{B}^{2} T}{3|e|} \frac{\partial \ln \sigma}{\partial E}
$$

Here, $\sigma$ is the conductivity, and $S$ is proportional to the derivative of $\ln \sigma$ with respect to the energy of the carriers near the Fermi level, denoted by $E$.

For graphene featured by linear dispersion relation near Dirac points, its conductivity is given by ref 20 :

$$
\sigma=\sigma_{\min }\left(1+\frac{E^{2}}{\Delta^{2}}\right)
$$




$$
E=\alpha \operatorname{sgn}\left(V_{g}-V_{D}\right) \sqrt{C_{g}\left(V_{g}-V_{D}\right)}
$$

Here $\sigma_{\min }$ is the minimum conductivity determined by the experiment, and the $\Delta$ is the width of the neutrality region of the graphene, $C_{g}$ denotes the capacitance per electron coupled with the gate. $V_{g}$ and $V_{D}$ represent the applied voltage of the gate and Dirac point voltage.

Thus, the Seebeck coefficient can be derived as a function with respect to voltage we apply on the gate, $V_{g}$, which is:

$$
S\left(V_{g}\right)=-\frac{\pi^{2} k_{B}^{2} T}{3|e|} \frac{1}{R} \frac{\partial R}{\partial V_{g}} \frac{\partial V_{g}}{\partial E}=\frac{\pi^{2} k_{B}^{2} T}{3|e| \alpha \sqrt{C_{g} \Delta^{\prime}}} \frac{2 \operatorname{sgn}\left(V_{g}-V_{D}\right)\left(\frac{V g-V_{D}}{\Delta^{\prime}}\right)^{\frac{1}{2}}}{1+\left(\frac{V g^{-}-V_{D}}{\Delta^{\prime}}\right)^{2}}
$$

where $\Delta^{\prime}$ is defined as $\frac{\Delta^{2}}{\alpha^{2} C_{g}}$, which is the key parameter in our graphene p-n junction device. By taking the derivative of $\frac{2 \operatorname{sgn}\left(V_{g}-V_{D}\right)\left(\frac{V_{g}-V_{D}}{\Delta^{\prime}}\right)^{\frac{1}{2}}}{1+\left(\frac{V g-V_{D}}{\Delta^{\prime}}\right)^{2}}$ with respect to $V_{g}$, we can get the points where the seebeck coefficient reaches either minimum or maximum. The distance between the max and min is $2 \mathrm{k} \Delta^{\prime}$, where $\mathrm{k} \in(1,2)$.

Since the PTE voltage is given by:

$$
V_{P T E}=\left(S_{1}-S_{2}\right) \Delta T=\frac{\pi^{2} k_{B}^{2} T \Delta T}{3|e| \alpha \sqrt{C_{g} \Delta^{\prime}}} \mathrm{f}\left(V_{g}\right)
$$

where $f\left(V_{g}\right) \triangleq \frac{2 \operatorname{sgn}\left(V_{g}-V_{D 1}\right)\left(\frac{V_{g}-V_{D 1}}{\Delta^{\prime}}\right)^{\frac{1}{2}}}{1+\left(\frac{V_{g}-V_{D 1}}{\Delta^{\prime}}\right)^{2}}-\frac{2 \operatorname{sgn}\left(V_{g}-V_{D 1}\right)\left(\frac{V_{g}-V_{D 2}}{\Delta^{\prime}}\right)^{\frac{1}{2}}}{1+\left(\frac{V_{g}-V_{D 2}}{\Delta^{\prime}}\right)^{2}}$, and $\Delta T$ is the temperature difference caused by the power of the laser spot.

It should be noted that we do not change the chemical potential difference along the p-n junction when we vary the voltage applied on the gate, i.e. $V_{D 1}$ and $V_{D 2}$ remain unchanged. By sweeping $V_{g}, S_{1}-S_{2}$ varies and reaches its maximum at a certain $V_{g}$, and we denote the maximum of $S_{1}-S_{2}$, (i.e. the maximum of $V_{P T E}$ when $\Delta T$ is considered as a constant) as $V_{\max }$. Clearly, $V_{\max }$ is maximized when $\left|V_{D 1}-V_{D 2}\right|=$ 
$2 \mathrm{k} \Delta^{\prime}$. This is how Fig. $4 \mathrm{e}$ is derived and accords well with the result of the experiment, which provides evidence of PTE dominance of photoresponse in our graphene p-n junctions.

The PTE effect is enhanced for the reason that the hot carriers heated by the laser in graphene tend to relax its heat to their cooler brothers rather than to the lattice, despite the fact that the latter is a common way for most semiconductors. The opposite signs of Seebeck coefficients between p-doped and n-doped areas of graphene further promote the PTE.

Next, let us examine the PV effect in our graphene p-n junctions and qualitatively determine to what extent we can neglect this impact of this conventional mechanism of photovoltage generation. The chemical potential difference between the $\mathrm{p}$ - and ntype domains gives rise to the built-in electric field, which drives the carriers with positive and negative charges along opposite directions, thus results in photovoltaic effect.

The current density could be written by:

$$
\boldsymbol{j}=\mathrm{e} \mu \bar{n} \nabla V_{g}
$$

where $\mu$ is the carrier mobility of the graphene sheet, and $\bar{n}$ is the density of photo-excited carriers.

By integrating the current density within the whole graphene sheet, we can derive the photovoltage resulted from PV effect as following:

$$
V_{\mathrm{PV}}=\frac{1}{W} \iint \sigma^{-1}(\mathbf{r}) \mathrm{e} \mu \bar{n}(\mathbf{r}) \nabla V_{g}(\mathbf{r}) \mathrm{d} x \mathrm{~d} y
$$

where $W$ is the width of the rectangle-shaped graphene.

Replace the $\sigma$ and $V_{g}$ by eq. (2)(3), we can get:

$$
V_{\mathrm{PV}}=\frac{\mu \alpha \bar{n} \sqrt{C_{g} \Delta^{\prime}}}{\sigma_{\min }}\left(\tan ^{-1} \sqrt{\frac{\left|V_{g}-V_{D 1}\right|}{\Delta^{\prime}}}-\tan ^{-1} \sqrt{\frac{\left|V_{g}-V_{D 2}\right|}{\Delta^{\prime}}}\right) \triangleq \frac{\mu \alpha \bar{n} \sqrt{C_{g} \Delta^{\prime}}}{\sigma_{\min }} g\left(V_{g}\right)
$$


Simply comparing the strength of the photovoltage caused by $V_{\mathrm{PTE}}$ and $V_{\mathrm{PV}}$, we have:

$$
\frac{V_{\mathrm{PTE}}}{V_{\mathrm{PV}}}=\frac{\pi^{2} k_{B}^{2} T \Delta T \sigma_{\min }}{3|e| \alpha^{2} \bar{n} \mu C_{g} \Delta^{\prime}} \frac{f\left(V_{g}\right)}{g\left(V_{g}\right)}
$$

To estimate $\Delta T$, we should resort to the temperature profile caused by the energy influx of the laser spot. Referring to ref. 6, the monochromatic laser spot with photon energy $\varepsilon_{0}$ and spot size $l_{0}$ gives $\Delta T$ by $\Delta T=\frac{\alpha \varepsilon_{0} l_{0} \dot{N} L}{\kappa}$, where $\dot{N}$ is the areal density of photon flux absorbed in the laser spot, L, the length of the graphene sheet, $\kappa$ the thermal conductivity coefficient. Replace $T$ by $\frac{3 \mathrm{e}^{2} \kappa}{\pi^{2} \mathrm{k}_{\mathrm{B}}^{2} \sigma}$ according to the Wiedemann-Franz relation, and $\bar{n}$ by $2 \tau_{0} \dot{N}$ with $\tau_{0}$ the carrier lifetime, we can achieve the proportion of $V_{\mathrm{PTE}}$ and $V_{\mathrm{PV}}$ :

$$
\frac{V_{\mathrm{PTE}}}{V_{\mathrm{PV}}}=\frac{e \varepsilon_{0} l_{0} L}{\alpha \mu \tau_{0} C_{g} \Delta^{\prime}} \frac{f\left(V_{g}\right)}{g\left(V_{g}\right)}
$$

Based on the real condition, we set $\varepsilon_{0}=2.3 \mathrm{eV}, l_{0}=1 \mu \mathrm{m}, \mathrm{L}=7 \mu \mathrm{m}, \tau_{0}=1 \mathrm{ps}$, $\Delta=\alpha \sqrt{C_{g} \Delta^{\prime}} \sim 200 \mathrm{meV}, \mu \sim 10000 \mathrm{~cm}^{2} \mathrm{~s}^{-1} \mathrm{~V}^{-1}$, we arrive at the rough comparison of the $V_{\mathrm{PTE}}$ and $V_{\mathrm{PV}}$ :

$$
\frac{V_{\mathrm{PTE}}}{V_{\mathrm{PV}}} \sim 10^{4} \frac{f\left(V_{g}\right)}{g\left(V_{g}\right)}
$$

The profile of $\frac{f\left(V_{g}\right)}{g\left(V_{g}\right)}$ is shown in Fig. $4 \mathrm{~d}$ in the text. Thus the impact of $V_{\mathrm{PV}}$ can be reasonably neglected. It should also be noted that PV effect will be further quenched if mobility of the graphene p-n junction decreases.

\section{Reference}

(1) Yan, K.; Wu, D.; Peng, H.; Jin, L.; Fu, Q.; Bao, X.; Liu, Z. Nat. Commun. 2012, 3,1280 . 
(2) Yu, Y.-J.; Zhao, Y.; Ryu, S.; Brus, L. E.; Kim, K. S.; Kim, P. Nano Lett. 2009, 9, 3430.

(3) Chiu, H.-Y., Perebeinos, V., Lin, Y.-M.; Avouris, P. Nano Lett. 2010, 10, 4634.

(4) Lohmann, T., von Klitzing, K.; Smet, J. H. Nano Lett. 2009, 9, 1973.

(5) Yu, T., Liang, C.-W., Kim, C.; Yu, B. Appl. Phys. Lett. 2011, 98, 243105.

(6) Peters, E. C., Lee, E. J., Burghard, M.; Kern, K. Appl. Phys. Lett. 2010, 97, 193102.

(7) Wang, H.; Zhou, Y.; Wu, D.; Liao, L.; Zhao, S.; Peng, H.; Liu, Z. Small 2013, 9, 1316-20.

(8) Koós, A.; Murdock, A.; Nemes-Incze, P.; Nicholls, R.; Pollard, A.; Spencer, S.; Shard, A.; Roy, D.; Biró, L.; Grobert, N., Phys. Chem. Chem. Phy. 2014, 16, 19446.

(9) Reddy, A. L. M.; Srivastava, A.; Gowda, S. R.; Gullapalli, H.; Dubey, M.; Ajayan, P. M. ACS Nano 2010, 4, 6337-6342.

(10)Panchakarla, L.; Subrahmanyam, K.; Saha, S.; Govindaraj, A.; Krishnamurthy, H.; Waghmare, U.; Rao, C. Adv. Mater. 2009, 21, 4726-4730.

(11) Zhong, J.; Deng, J.-J.; Mao, B.-H.; Xie, T.; Sun, X.-H.; Mou, Z.-G.; Hong, C.-H.; Yang, P.; Wang, S.-D. Carbon 2012, 50, 335-338.

(12) Xu, X.; Gabor, N. M.; Alden, J. S.; van der Zande, A. M.; McEuen, P. L. Nano 
Lett. 2009, 10, 562-566.

(13) Gabor, N. M.; Song, J. C.; Ma, Q.; Nair, N. L.; Taychatanapat, T.; Watanabe, K.; Taniguchi, T.; Levitov, L. S.; Jarillo-Herrero, P. Science 2011, 334, 648-652.

(14) Xia, F.; Mueller, T.; Golizadeh-Mojarad, R.; Freitag, M.; Lin, Y.-m.; Tsang, J.; Perebeinos, V.; Avouris, P. Nano Lett. 2009, 9, 1039-1044.

(15) Song, J. C. W.; Rudner, M. S.; Marcus, C. M.; Levitov, L. S. Nano Lett. 2011, 11, 4688-4692.

(16) Freitag, M.; Low, T.; Xia, F. N.; Avouris, P., Nat. Photonics 2013, 7, 53-59.

(17) Lemme, M. C.; Koppens, F. H.; Falk, A. L.; Rudner, M. S.; Park, H.; Levitov, L. S.; Marcus, C. M. Nano Lett. 2011, 11, 4134-4137.

(18) Peters, E. C.; Lee, E. J. H.; Burghard, M.; Kern, K. Appl. Phys. Lett. 2010, 97 193102.

(19) Ashcroft, N. W. \& Mermin, N. D. in Solid state phys Ch.32 (Saunders College, Philadelphia, 1976).

(20) Castro Neto, A. H., Guinea, F., Peres, N. M. R., Novoselov, K. S. \& Geim, A. K. The electronic properties of graphene. Rev. Mod. Phys. 2009, 81, 109-162. 